INPLASY

PROTOCOL

To cite: Calmon et al. Is

bruxism a risk factor for noncarious cervical lesions?

Inplasy protocol 202190040. doi:

10.37766/inplasy2021.9.0040

Received: 13 September 2021

Published: 13 September 2021

Corresponding author:

Kezia Calmon

keziacalmon@gmail.com

Author Affiliation:

State University of Rio de Janeiro

\section{Support: CAPES.}

Review Stage at time of this submission: Formal screening of search results against eligibility criteria.

\section{Is bruxism a risk factor for non- carious cervical lesions?}

Calmon, K1; Foscaldo, T2; Vieira, L3; Perez, C4; Senna, P5; Santos, APP6.

Review question / Objective: Do patients with bruxism have a higher risk of developing non-carious cervical lesions than patients who do not have bruxism? Thus, the PECOs approach is: $(P)$ Population/problem: Patients from 13 years old; (E) Exposition: bruxism; (C) Comparison: Patients without bruxism; (O) Outcome: non-carious cervical lesions; (S) Study Type: Observational studies (Cohort, Case Control and CrossSectional).

Condition being studied: The term "non-carious cervical lesion" has been used to describe cervical tooth wear created through different processes that are not associated with dental caries. Bruxism is a repetitive masticatory muscle activity characterized by clenching or grinding of the teeth and/or by bracing or thrusting of the mandible.

INPLASY registration number: This protocol was registered with the International Platform of Registered Systematic Review and Meta-Analysis Protocols (INPLASY) on 13 September 2021 and was last updated on 10 November 2021 (registration number INPLASY202190040).

Conflicts of interest:

None declared.

\section{INTRODUCTION}

Review question / Objective: Do patients with bruxism have a higher risk of developing non-carious cervical lesions than patients who do not have bruxism? Thus, the PECOs approach is: (P) Population/problem: Patients from 13 years old; (E) Exposition: bruxism; (C)
Comparison: Patients without bruxism; (0) Outcome: non-carious cervical lesions; (S) Study Type: Observational studies (Cohort, Case Control and Cross-Sectional).

Rationale: Although bruxism is commonly associated with dental wear, there is no scientific consensus that bruxism alone can be considered an etiological factor of 
non-carious cervical lesions. In the last two decades, some studies have found a positive association between this type of lesions and occlusal forces and bruxism. However, there are still some studies that report contradictory or inconclusive results. Therefore, we aim to search on the literature all the clinical studies that evaluated the presence of non-carious cervical lesions in patients with bruxism.

Condition being studied: The term "noncarious cervical lesion" has been used to describe cervical tooth wear created through different processes that are not associated with dental caries. Bruxism is a repetitive masticatory muscle activity characterized by clenching or grinding of the teeth and/or by bracing or thrusting of the mandible.

\section{METHODS}

Search strategy: An electronic search will be independently conducted to identify potential studies. The following databases will be screened: MEDLINE/PubMed, EMBASE, Web of Science, LILACS and BBO/Virtual Health Library, and the grey literature (Open Grey). A combination of MeSH/DECs, synonyms, and free terms with the Boolean operators OR and AND will be used as follows: (ltooth erosion[MeSH Terms]) OR (tooth abrasion[MeSH Terms])) OR (tooth wear[MeSH Terms])) OR (tooth attrition[MeSH Terms])) OR (tooth erosion[Title/Abstract])) OR (dental erosion[Title/Abstract])) OR (tooth abrasion [Title/Abstract])) OR (dental abrasion [Title/Abstract])) OR (abfraction[Title/Abstract])) OR (abfractionlike[Title/Abstract])) OR (dental cervical lesions[Title/Abstract])) OR (worn dentition[Title/Abstract])) OR (tooth surface lesions[Title/Abstract])) OR (tooth loss defects[Title/Abstract])) OR (tooth wear[Title/Abstract])) OR (noncarious cervical[Title/Abstract])) OR (non carious cervical[Title/Abstract])) OR (non-carious cervical[Title/Abstract])) OR (tooth surface loss[Title/Abstract])) OR (tooth loss defects[Title/Abstract])) OR (tooth tissue loss[Title/Abstract])) OR (occlusal
wear[Title/Abstract])) OR (dental wear[Title/ Abstract])) OR (NCCL*[Title/Abstract])) OR (tooth attrition[Title/Abstract])) OR (dental attrition [Title/Abstract])) AND ()((广()((bruxism[MeSH Terms]) OR (sleep bruxism[MeSH Terms])) OR (bruxism[Title/ Abstract])) OR (sleep bruxism[Title/ Abstract])) OR (occlusal splint*[Title/ Abstract])) OR (teeth grinding disorder*[Title/Abstract])) OR (bruxomania[Title/Abstract])) OR (oral parafunction *[Title/Abstract])) OR (parafunctional activit*[Title/Abstract])) OR (parafunctional habit*[Title/Abstract])) OR (sleep disorder ${ }^{\star}[$ Title/Abstract])).

Participant or population: Patients from 13 years old.

Intervention: Bruxism.

Comparator: Patients without bruxism.

Study designs to be included: Observational studies (cross-sectional, cohort and casecontrol)

Eligibility criteria: Observational studies that evaluated the presence of non-carious cervical lesions and bruxism in patients from 13 years old.

Information sources: We aim to search on electronic databases (MEDLINE/PubMed, EMBASE, Web of Science, LILACS and BBO/Virtual Health Library), grey literature (Open Grey), studies registers (PROSPERO, INPLASY), conference papers (International Association for Dental Research - IADR), and to perform a direct search in references of selected articles.

Main outcome(s): The presence of noncarious cervical lesions, irrespective of the diagnostic criteria used.

Data management: We will manage records with the Mendeley Reference Management Software. The studies selection and extraction of data will be performed by two independent reviewers, KC and TF. Any disagreement will be resolved, if necessary, by a third author (LC). 
Quality assessment / Risk of bias analysis: For the quality assessment we will use The Joanna Briggs Institute tools (JBI).

Strategy of data synthesis: Grouping data will be based on study design, population characteristics and studies' risk measures (relative risk and odds ratio). Descriptive summaries of studies will be entered into tables and a narrative synthesis of evidence will be performed. A summary measure will be calculated using a randomeffects model. Meta-analysis will not be conducted if clinical heterogeneity is high.

Subgroup analysis: If data permits, we will perform a subgroup analysis: sleep bruxism $x$ awake bruxism.

Sensitivity analysis: If sufficient available data are extracted, we will conduct sensitivity analysis to check the stability for the outcome results by excluding studies with high risk of bias.

Language: No language limits will be imposed on the search.

Country(ies) involved: Brazil.

Keywords: systematic review, bruxism, tooth wear, tooth abrasion.

Contributions of each author:

Author 1 - Kezia Calmon - Project, search strategy, study selection, data extraction, description of results, and article writing.

Email: keziacalmon@gmail.com

Author 2 - Tatiana Foscaldo - Project, search strategy, study selection, data extraction, description of results, and article writing.

Author 3 - Lanna Vieira - Project, search strategy, study selection, data extraction, description of results, and article writing.

Author 4 - Cesar Perez - Project, search strategy, description of results, data analysis, reviewing the article.

Author 5 - Plinio Senna - Project, search strategy, description of results, data analysis, reviewing the article.

Author 6 - Ana Paula Pires dos Santos Project, data extraction, description of results, article writing, and reviewing the article. 